\title{
Numerical solution of Fisher's equation using finite difference
}

\author{
Dr. Sharefa Eisa Ali Alhazmi \\ Umm Al-Qura University College of Education for Girls at Al-Qunfudah \\ Mathematics department, Macca, KSA \\ E-mail: sehazmi@uqu.edu.sa
}

\begin{abstract}
Keywords: Non-Linear Diffusion Equation, Integral equation, Fisher's equation, Forward time centered space, Crank Nicholson method.
\end{abstract}

\begin{abstract}
A numerical method is proposed to approximate the numeric solutions of nonlinear Fisher's reaction diffusion equation with finite difference method. The method is based on replacing each terms in the Fisher's equation using finite difference method. The proposed method has the advantage of reducing the problem to a nonlinear system, which will be derived and solved using Newton method. FTCS and CN method will be introduced, compared and tested.
\end{abstract}

\section{Introduction}

The Fisher equation arises in heat and mass transfer, biology, and ecology. It's introduced to describe the spreading of genes [1] has found applications in different fields of research ranging from ecology [2] to plasma physics [3]. Lie symmetry method is applied to analyze Fisher's equation in cylindrical coordinates. Symmetry algebra is found and symmetry invariance is used to reduce the equation to a first order ODE. The first order ODE is further analyzed to obtain exact solution of Fisher's equation in explicit form. In this paper we will not investigate the theoretical methods how to solve the Fisher's equation. Let the integral equation

$$
\begin{aligned}
u(t, x) & =f(x)+\int_{0}^{t} \alpha(\tau, x) \frac{\partial^{2}}{\partial x^{2}} u(\tau, x) d \tau+\int_{0}^{t} \mathcal{F}(u(\tau, x)) d \tau \\
u(t, L) & =u(t,-L)=0
\end{aligned}
$$

where $F$ is a given (presumably nonlinear) function of $u(t, x), f(x)$ is also a given function, $L$ is a constant and _ $(t, x)$ is is the diffusion function. We take $t$ as a time variable and $x \in[-L, L]$. This problem can be transformed to nonlinear PDEs with Dirichlet boundary conditions and exactly we are going to solve the following problem:

$$
\begin{array}{rll}
\text { PDE }: \frac{\partial}{\partial t} u(t, x) & = & \alpha(t, x) \frac{\partial^{2}}{\partial x^{2}} u(t, x)+\mathcal{F}(u(t, x)) \\
\text { Boundary condition }: u(t, L) & = & u(t,-L)=0 \\
\text { Initial condition : } u(0, x) & =f(x) &
\end{array}
$$

In the case where F is linear the PDE can an be solved by the separation of variables methods. Here, is the diffusion constant. This is a parabolic, nonlinear and nonhomogeneous PDE in two dimensions. In this equation, $t$ plays the role of a time variable and $\mathrm{x}$ is a spatial variable; that is, we are going to solve the PDE as an initial value problem. In order to obtain a numeric solution, we will need to supplement the PDE with boundary and initial conditions. However if, as in many of the applications considered in [4], $\mathrm{F}$ is nonlinear function then the problem is much more burdensome. It is not usually possible to obtain general analytical traveling wave solutions and one must analyze such problems numerically. Spectral methods (see, for instance, $[7,8,9,10,11]$ ) are powerful techniques that we use to numerically solve linear and nonlinear partial differential equations either in their strong or weak forms. Legendre Spectral Collocation Method is used to solve the Fisher's equation, see [12]. In [13], the author solve the Fisher's equation using PetrovGalerkin finite element method. In [14], some numerical study of Fisher's equation by Adomian's method was proposed. In [15], the author study a nonlinear reactiondiffusion equation for its 
traveling waves. These methods look very difficult. For this reason, we will focus on a nonlinear PDEs using finite difference methods and we restrict our work in the case where the function_( $t, x)$ $=$ _ constant. This method used in this paper can be applied for any nonlinear function, but here we limit our presentation to the case of Fisher's equation which corresponding to the choice of $\mathrm{F}(\mathrm{u})=$ $\mathrm{u}(1-\mathrm{u})$. It was suggested by Fisher as a deterministic version of a stochastic model for the spatial spread of a favored gene in a population. The rest of this paper is organized as follows:In section 2, we present the two method FTCS and CN. In ??, we solve the Fisher's equation using FTSC and we look to it's stability and some remarks about maximum principle will be presented. Section 4 , give a comparison between the FTCS and CN methods and their stability.

Finally, we conclude.

\section{Approximation}

The key step in solving our Fisher's equation numerically using finite difference methods is to replace the derivatives with so-called "finite difference method.

Forward time Method: $\quad \frac{\partial}{\partial t} u(t, x)=-\frac{u(t, x)}{s}+\frac{u(t+s, x)}{s}$

Backward time Method: $\frac{\partial}{\partial t} u(t, x)=-\frac{\stackrel{s}{u(t-s, x)}}{s}+\frac{u(t, x)}{s}$

Centered space : $\frac{\partial^{2}}{\partial x^{2}} u(t, x)=\frac{u(t, x-h)}{h^{2}}-2 \frac{u(t, x)}{h^{2}}+\frac{u(t, x+h)}{h^{2}}-\frac{1}{12}\left(\frac{\partial^{4}}{\partial x^{4}} u(t, x)\right) h^{2}$

Note that the last term on the RHS of previous approximations is just the leading order term in the error; terms with higher powers of $h$ have been omitted. The power of $h$ in the leading error term is called the order of the method. One can give better approximation using more points in previous approximations.

The FTCS (Forward time Centered space) is obtained by putting forward time and centered space approximation into the PDE, we get:

$$
-\frac{u(t, x)}{s}+\frac{u(t+s, x)}{s}=\alpha\left(\frac{u(t, x-h)}{h^{2}}-2 \frac{u(t, x)}{h^{2}}+\frac{u(t, x+h)}{h^{2}}\right)+\mathcal{F}(u(t, x))
$$

The BTCS method is obtained by putting backward time and centered space method into the PDE:

$$
-\frac{u(t, x)}{s}+\frac{u(t+s, x)}{s}=\alpha\left(\frac{u(t+s, x-h)}{h^{2}}-2 \frac{u(t+s, x)}{h^{2}}+\frac{u(t+s, x+h)}{h^{2}}\right)+\mathcal{F}(u(t+s, x))
$$

The Crank-Nicholson method is the average of the FTCS and BTCS:

$$
\begin{aligned}
-\frac{u(t, x)}{s}+\frac{u(t+s, x)}{s} & =\frac{1}{2} \alpha\left(\frac{u(t, x-h)}{h^{2}}-2 \frac{u(t, x)}{h^{2}}+\frac{u(t, x+h)}{h^{2}}\right)+\frac{1}{2} \mathcal{F}(u(t, x)) \\
& +\frac{1}{2} \alpha\left(\frac{u(t+s, x-h)}{h^{2}}-2 \frac{u(t+s, x)}{h^{2}}+\frac{u(t+s, x+h)}{h^{2}}\right) \\
& +\frac{1}{2} \mathcal{F}(u(t+s, x))
\end{aligned}
$$

The method used is "centered" because $u$ is evaluated at an equal number of points to the right and left of the point where we want to approximate the derivative. Fig 1, a configuration of the FTCS and $\mathrm{CN}$ methods. The Crank-Nicholson method relates three future values to three past values.

This means that, as for the BTCS method is an undetermined equation for the future field values if the past values are known. Here we ask the following question: why bother with method such as BTCS or Crank-Nicholson (which involve the solution of a potentially large system of equations at each time step) over the simpler FTCS method? The answer is the stability of the various schemes. Let's compare the output from the FTCS and Crank-Nicholson schemes in the next section. 

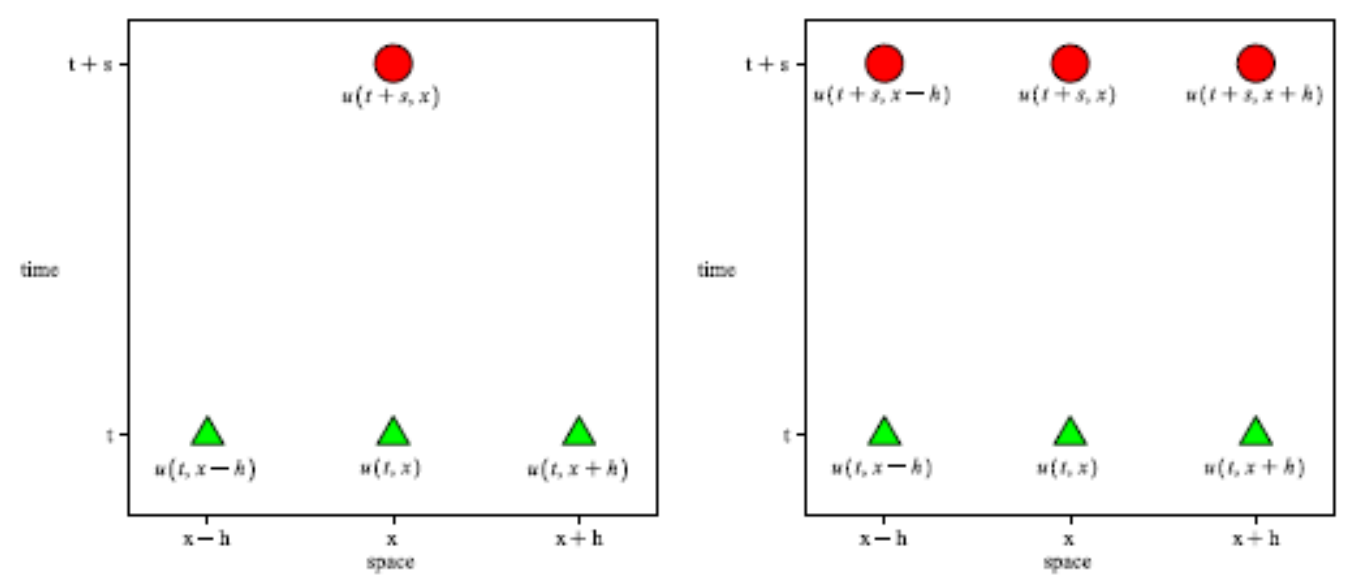

Figure 1: Information flow in the FTCS method and CN method

\section{Forward time centered-space solution}

The time derivative will be replaced with a "forward time" right-oriented method with 2 point. The "forward time" label comes from the fact that we are approximating the time derivative at $\mathrm{u}(\mathrm{t}, \mathrm{x})$ using a point to the future. Notice that we have denoted the step-size in the time direction by s. The spatial derivative will be approximated with a centered 3 point stencil with step-size $h$. The FTCS stencil can be solved for $\mathrm{u}(\mathrm{t}+\mathrm{s}, \mathrm{x})$ for any form of the potential $\mathrm{F}(\mathrm{u}(\mathrm{t}, \mathrm{x}))$ :

$$
u(t+s, x)=\frac{s \alpha u(t, x-h)}{h^{2}}-2 \frac{s \alpha u(t, x)}{h^{2}}+\frac{s \alpha u(t, x+h)}{h^{2}}+s \mathcal{F}(u(t, x))+u(t, x)
$$

We can always calculation the solution $\mathrm{u}(\mathrm{t}, \mathrm{x})$ at a future time step with knowledge of the field at a previous time step, irregardless of the nature of the nonlinearity in the potential F. This means that the numerical FTCS algorithm to solve our problem is virtually the same as it is for the linear equation. We introduce, some parameter will be used in our method:

$$
\begin{aligned}
& \tau=1, N=400, M=20, L=1, \alpha=1 \\
& X:=j \mapsto L\left(-1+2 \frac{j}{M+1}\right) \\
& T:=i \mapsto \frac{\tau i}{N}
\end{aligned}
$$$$
\text { Time step } s:=T(1)-T(0)=0.0952380952
$$$$
\text { Space step } h:=X(1)-X(0)=0.0025
$$$$
f(x)=\mathrm{e}^{-16 x^{2}}
$$

Figure 2 compare the numerical and the exact solution obtained using Maple. If the initial data $\mathrm{u}(0$, $\mathrm{x})=\mathrm{f}(\mathrm{x})$ is nonnegative then the solution of the Fisher's equation remains nonnegative, this is consistent with its use as a density of probability or density of population. Figure 2 show that the maximum principle is satisfied. Indeed, the maximum principle is verified because if the solution changes sign from negative to positive or vice versa at ( $\mathrm{t} 0, \mathrm{x} 0)$ and if the solution has a nondegenerate minimum at $\mathrm{x} 0$ then $\operatorname{uxx}(\mathrm{t} 0, \mathrm{x} 0)>0$. Therefore, the solution cannot involve forward in time into the region $\mathrm{u}<0$. A more careful argument is required to deal with degenerate minimum. So, a similar argument so that if the initial data $\mathrm{f}(\mathrm{x})<1$ then the solution remains less than one, i.e. $\mathrm{u}(\mathrm{t}, \mathrm{x})<1$. 


\section{Crank Nicholson solution}

Here, we denote future field values as: $j=u(t+s, x j)$ and past field values as $j=u(t, x j)$ Using

$$
\begin{aligned}
u(t+s, x-h) & =\phi_{j-1}, u(t+s, x)=\phi_{j}, u(t+s, x+h)=\phi_{j+1} \\
u(t, x-h) & =\psi_{j-1},(t, x)=\psi_{j}, u(t, x+h)=\psi_{j+1}
\end{aligned}
$$

Then we get

$$
-2 \phi_{j} h^{2}+\alpha s \phi_{j-1}-2 \alpha s \phi_{j}+\alpha s \phi_{j+1}+\mathcal{F}\left(\phi_{j}\right) s h^{2}=-2 h^{2} \psi_{j}-s \alpha \psi_{j-1}+2 s \alpha \psi_{j}-s \alpha \psi_{j+1}-s h^{2} \mathcal{F}\left(\psi_{j}\right)
$$

Here is an example of the nonlinear system of equations we have to solve at each time step for

$$
\begin{aligned}
& \left\{\phi_{j}\right\}_{j=1}^{M} \\
& -2 \phi_{j} h^{2}+\alpha s \phi_{j-1}-2 d s \phi_{j}+d s \phi_{j+1}+\mathcal{F}\left(\phi_{j}\right) s h^{2}=-2 h^{2} \psi_{j}-s \alpha \psi_{j-1}+2 s \alpha \psi_{j}-s \alpha \psi_{j+1}-s h^{2} \mathcal{F}\left(\psi_{j}\right)
\end{aligned}
$$

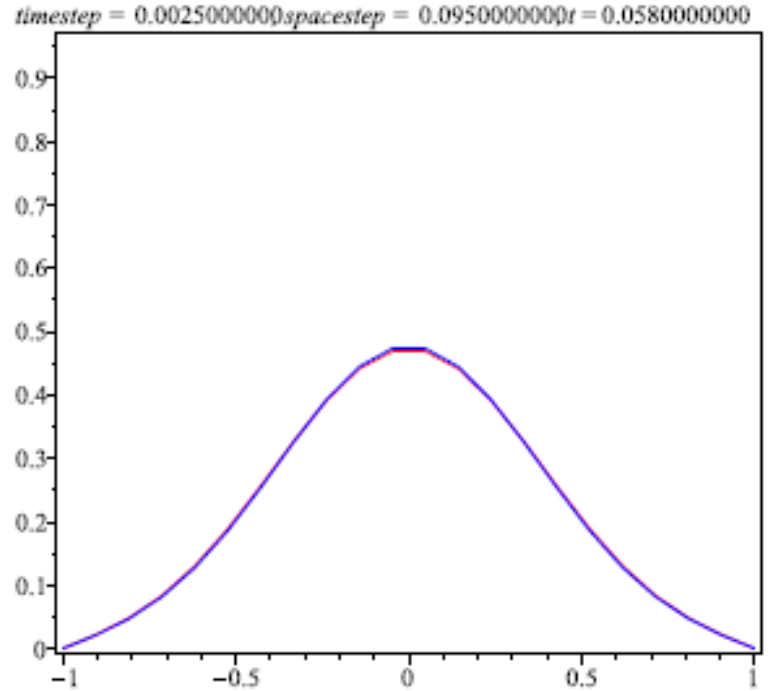

numerical solution (FTCS- pdsolve/numerig

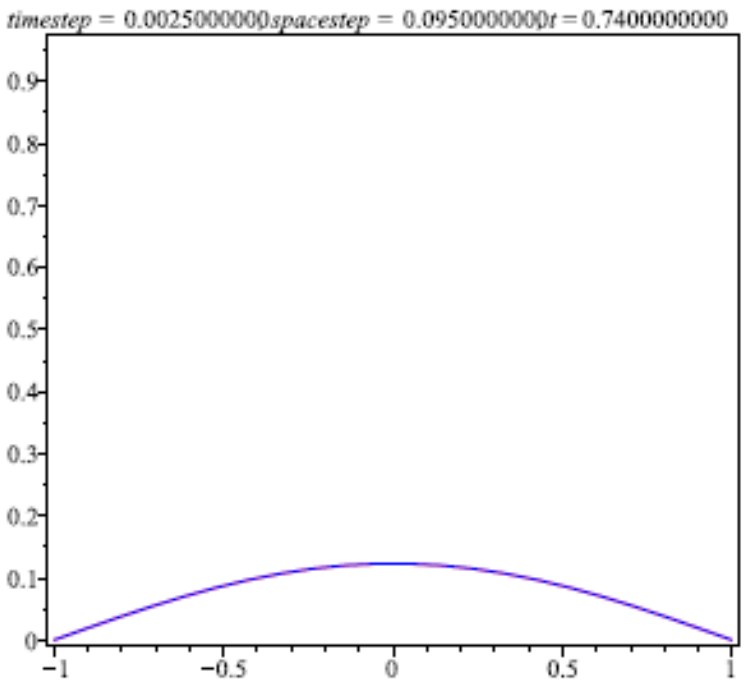

numerical solution (FTCS- pdsolve/numeric

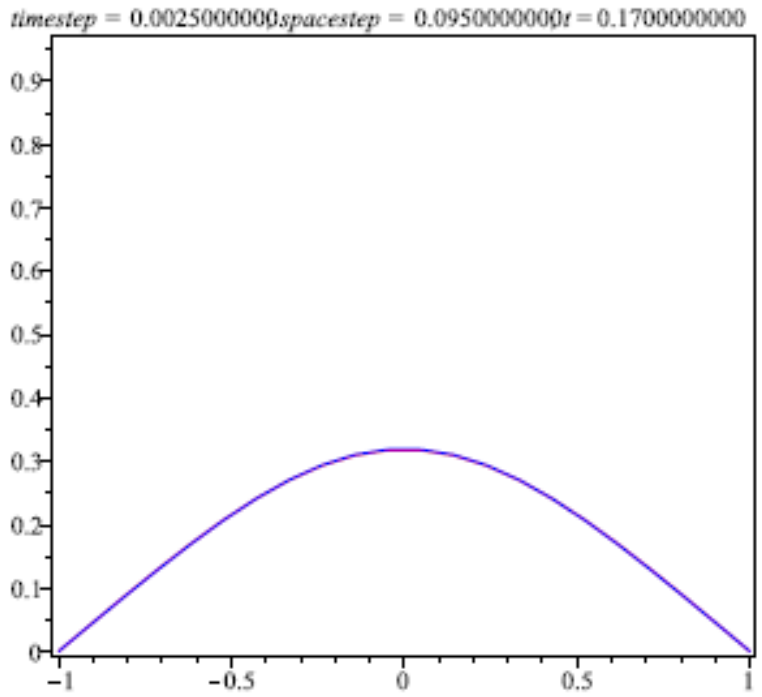

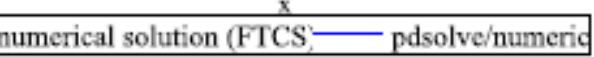

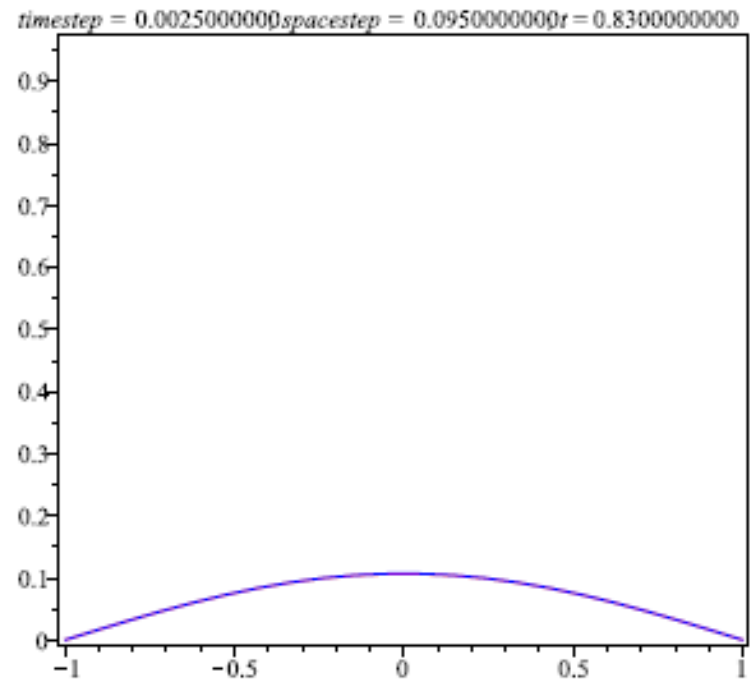

numerical solution (FTCS- pdsolve/numerid 

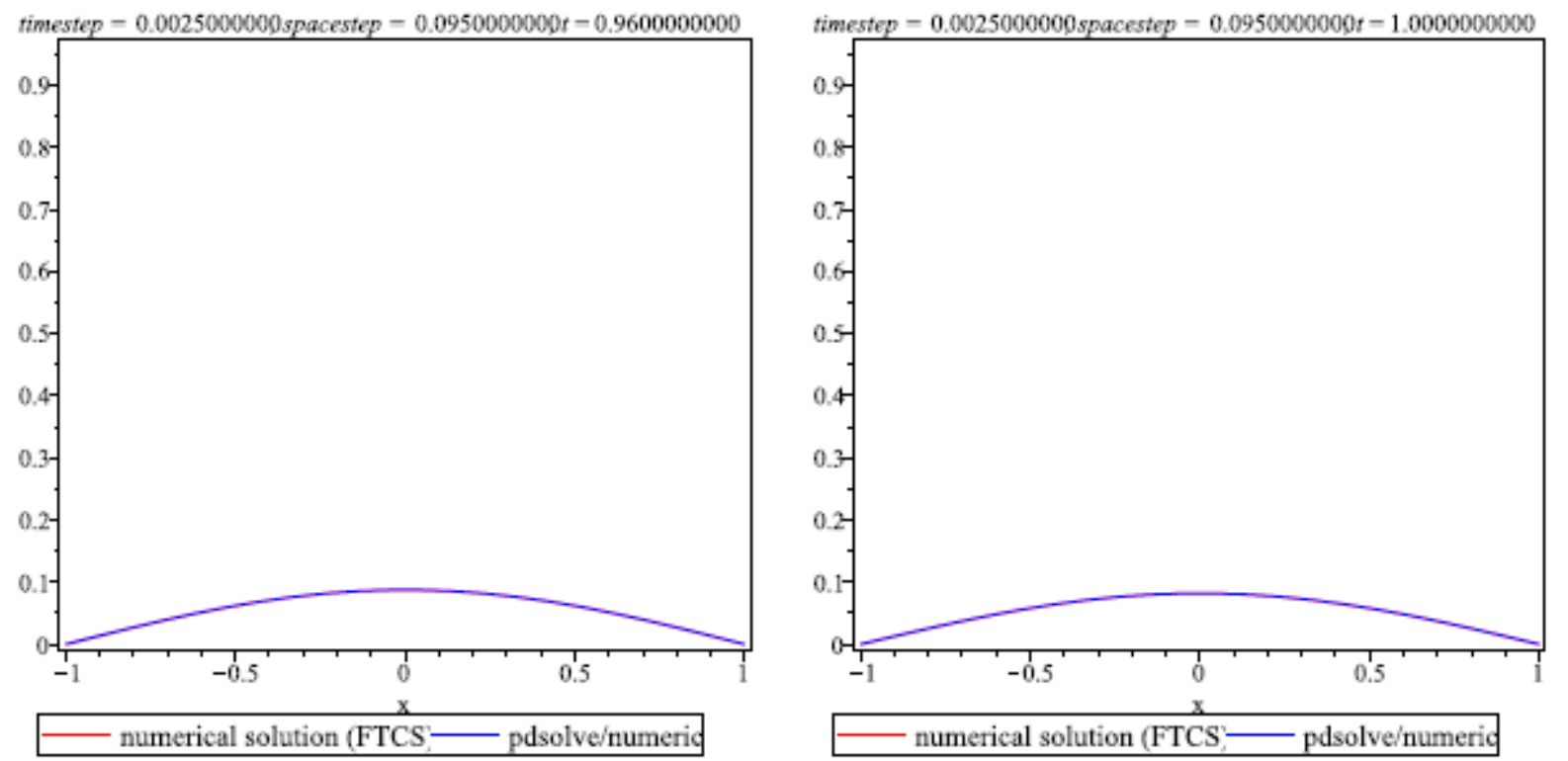

Figure 2: Comparison between FTCS method and the true solution at different time.

When $\mathrm{M}=5$ interior lattice points and the boundary conditions imply

$$
\phi_{0}=\phi_{M+1}=\psi_{0}=\psi_{M+1}=0
$$

We have the system with $\phi_{0}=\psi_{0}=\psi_{6}=\phi_{6}=0$

$$
\begin{aligned}
-2 \phi_{1} h^{2}-2 \alpha s \phi_{1}+\alpha s \phi_{2}+\mathcal{F}\left(\phi_{1}\right) s h^{2} & =-2 h^{2} \psi_{1}+2 s \alpha \psi_{1}-s \alpha \psi_{2}-s h^{2} \mathcal{F}\left(\psi_{1}\right) \\
-2 \phi_{2} h^{2}+\alpha s \phi_{1}-2 \alpha s \phi_{2}+d s \phi_{3}+\mathcal{F}\left(\phi_{2}\right) s h^{2} & =-2 h^{2} \psi_{2}-s \alpha \psi_{1}+2 s \alpha \psi_{2}-s \alpha \psi_{3}-s h^{2} \mathcal{F}\left(\psi_{2}\right) \\
-2 \phi_{3} h^{2}+\alpha s \phi_{2}-2 \alpha s \phi_{3}+\alpha s \phi_{4}+\mathcal{F}\left(\phi_{3}\right) s h^{2} & =-2 h^{2} \psi_{3}-s \alpha \psi_{2}+2 s \alpha \psi_{3}-s \alpha \psi_{4}-s h^{2} \mathcal{F}\left(\psi_{3}\right) \\
-2 \phi_{4} h^{2}+\alpha s \phi_{3}-2 \alpha s \phi_{4}+\alpha s \phi_{5}+\mathcal{F}\left(\phi_{4}\right) s h^{2} & =-2 h^{2} \psi_{4}-s \alpha \psi_{3}+2 s \alpha \psi_{4}-s \alpha \psi_{5}-s h^{2} \mathcal{F}\left(\psi_{4}\right) \\
-2 \phi_{5} h^{2}+\alpha s \phi_{4}-2 \alpha s \phi_{5}+\mathcal{F}\left(\phi_{5}\right) s h^{2} & =-2 h^{2} \psi_{5}-s \alpha \psi_{4}+2 s \alpha \psi_{5}-s h^{2} \mathcal{F}\left(\psi_{5}\right)
\end{aligned}
$$

Our system can be transformed to

$$
G_{i}\left(\phi_{1}, \phi_{2}, \phi_{3}, \phi_{4}, \phi_{5}\right)=0, i=1, \ldots, 5
$$

It is useful to organize the unknowns into a vector $\phi=\left[\phi_{\mathbf{1}}, \phi_{\mathbf{2}}, \phi_{\mathbf{3}}, \phi_{\mathbf{4}}, \phi_{\mathbf{5}}\right]^{\mathrm{t}}$ Then, we will linearize the problem by taking $\phi=\phi_{\mathbf{k}}+\delta_{\mathbf{k}}$ where $\phi_{\mathbf{k}}$ is a guess and $\delta_{k}$ is the error in the guess. Expanding $G_{i}(\phi)=G_{i}\left(\phi_{\mathbf{k}}+\delta_{\mathbf{k}}\right)=0$ to linear order in the error, we obtain:

$$
G\left(x_{k}\right)+J\left(x_{k}\right) \delta_{k}=0
$$

Where

$$
G\left(x_{k}\right)=\left[\begin{array}{c}
G_{1}\left(x_{k}\right) \\
G_{2}\left(x_{k}\right) \\
G_{3}\left(x_{k}\right) \\
G_{4}\left(x_{k}\right) \\
G_{5}\left(x_{k}\right)
\end{array}\right]
$$


The matrix $J(x k)$ is the Jacobian of the system. Then, the linear system (6) can be solved for $\delta_{k}$ which can then be used to update our guess via

$$
\mathrm{x}_{\mathrm{k}+1}=\mathrm{x}_{\mathrm{k}}+\delta_{\mathrm{k}}=\mathrm{x}_{\mathrm{k}}-\mathbf{J}^{-1}\left(\mathrm{x}_{\mathrm{k}}\right) \mathrm{G}\left(\mathrm{x}_{\mathrm{k}}\right)
$$

One can iterate this process until the distance between the iterates $\frac{\left|\mathbf{x}_{\mathbf{k}+\mathbf{1}}-\mathbf{x}_{\mathbf{k}}\right|}{n}$ is less than some user defined tolerance. This method is know as a Newton method. Using Newton method to solve nonlinear equations of this type in the process of creating the Crank Nicholson solution. Note that for sufficiently small time steps we expect ${ }^{\phi_{j}} \sim \psi_{j}$ so a good initial guess for the future field values are just the corresponding past field values.

$$
\begin{aligned}
& \tau=5, N=400, M=100, L=5, \alpha=1 \\
& X:=j \mapsto L\left(-1+2 \frac{j}{M+1}\right) \\
& T:=i \mapsto \frac{\tau i}{N}
\end{aligned}
$$

Time step $s:=T(1)-T(0)=0.0952380952$

Space step $h:=X(1)-X(0)=0.0025$

$$
f(x)=\mathrm{e}^{-100 x^{2}}
$$

For this choice the FTCS is unstable, see Fig 3 however CN method gives reasonable results that are closed to the exact solution, see Fig 4.
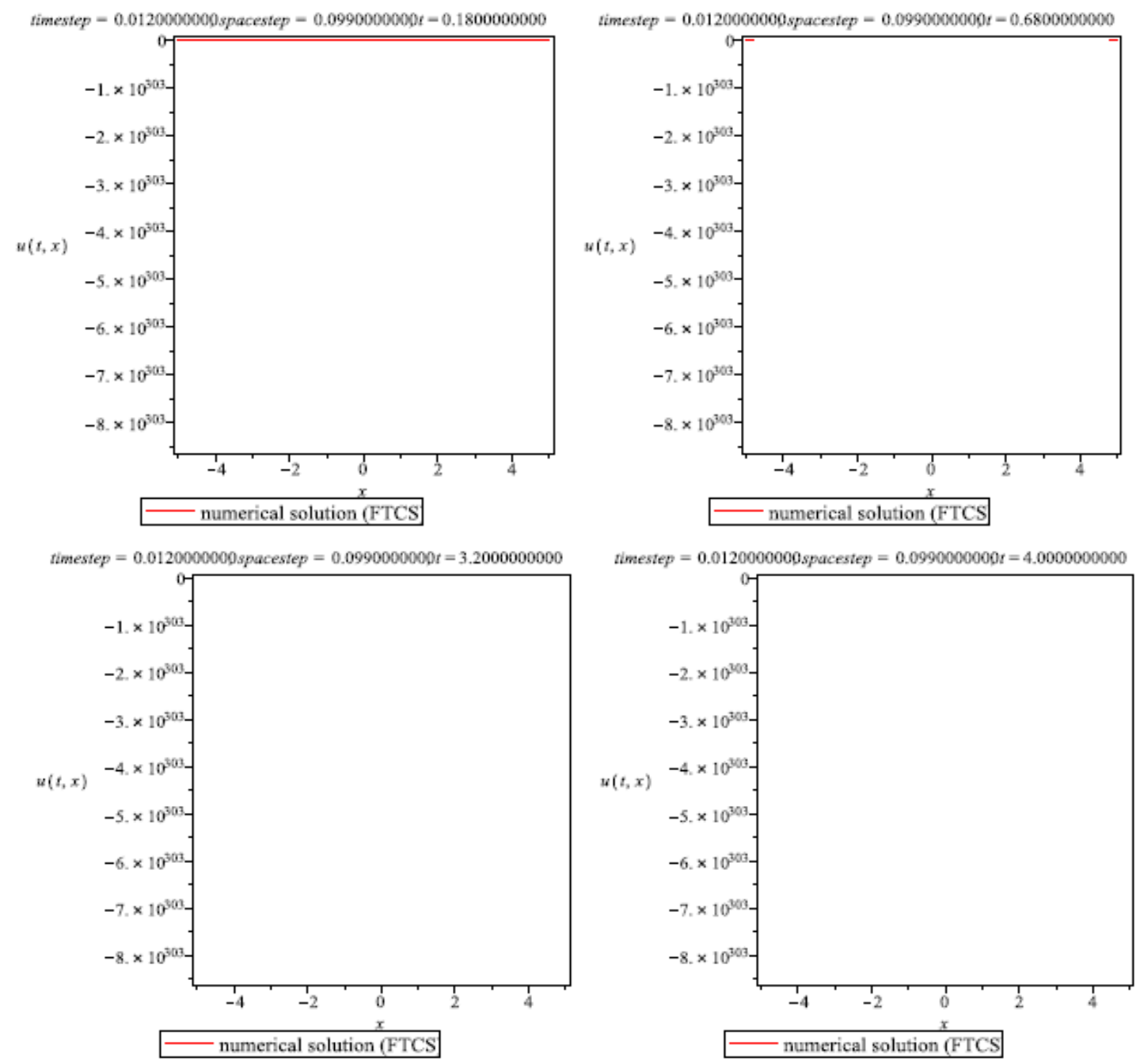

Figure 3: Comparison between FTCS method and the true solution at different time. 

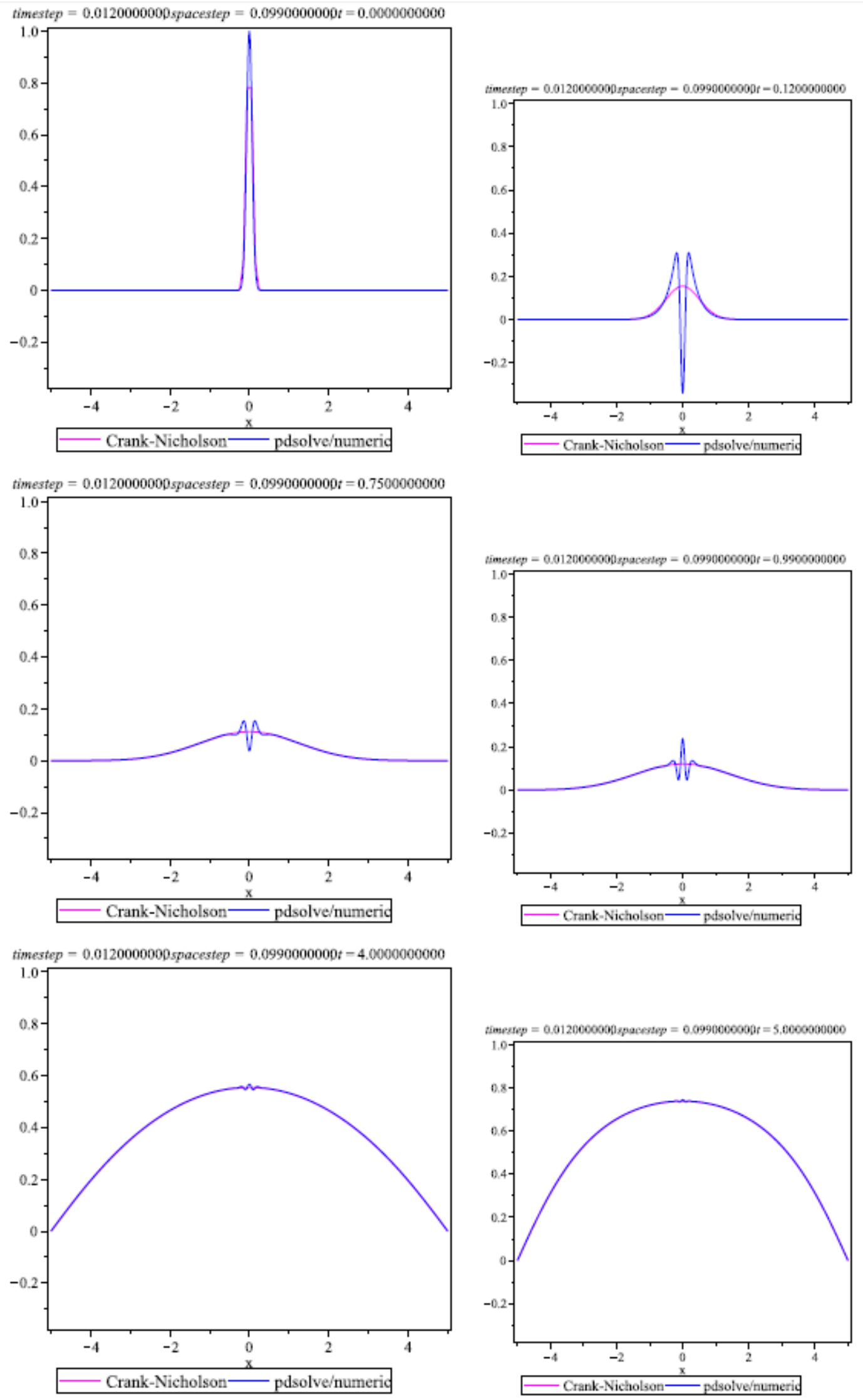

Figure 4: Comparison between $\mathrm{CN}$ method and the true solution at different time. 


\section{Conclusion}

Our numerical results can be applied for $\frac{\partial}{\partial t} u(t, x, y)=\Delta u(t, x, y)+\mathcal{F}(u(t, x, y))$ It's a good application of the strategy proposed in this work. The Crank-Nicolson method is more accurate than FTCS or BTCS. Clearly, something is going very wrong with the FTCS method, while the CN one is returning reasonable results. Experimenting with different $\mathrm{N}$ and $\mathrm{M}$ for the Crank- Nicholson method convince us that its output for $\mathrm{u}(\mathrm{t}, \mathrm{x})$ is always bounded and not exponentially diverging. Although all three methods have the same spatial truncation error $\mathrm{h} 2$ the better temporal truncation error for the Crank-Nicolson method is big advantage. The Crank-Nicolson scheme is recommended over FTCS and BTCS. One can seek a condition for unstable behavior of FTCS.

\section{References}

[1] R. A. Fisher. The Wave of Advance of Advantageous Genes, Annals of Genetics, Vol. 7, No.4, 1937, p. 353.

[2] A. Kolmogorov, I. Petrovskii and N. Piscounov. Etude de L'quation de la Diffusion Avec Croissance de la Quantit de Matire et Son Application a un Problem Biologique. In: V. M.Tikhomirov, Ed., Selected Works of A. N. Kolmogorov I, Kluwer, Dordrecht, 1991, p. 248.

[3] B. H. Gilding and R. Kersner. Travelling Waves in Nonlinear Diffusion Convection Reaction. Birkhuser, Basel, 2004. doi:10.1007/978-3-0348-7964-4.

[4] J. D. Murray. Mathematical Biology. Springer-Verlag, New York, 1993.

[5] R. A. Fisher. The wave of advance of advantageous genes. Ann. Eugenics, 7 (1937), 353369.

[6] M.J. Ablowitz and A. Zeppetella. Explicit solution of Fisher's equation for a special wave speed. Bull. Math. Biol., Vol. 41(835), (1979).

[7] D. A. Kopriva. Implementing Spectral Methods for Partial Differential Equations: Algorithms for Scientists and Engineers. Springer, Berlin, Germany, 2009.

[8] C. Canuto,M.Y.Hussaini, A. Quarteroni, and T. A. Zang. SpectralMethods: Fundamentals in Single Domains, Springer, Berlin, Germany, 2006.

[9] C. I. Gheorghiu. Spectral Methods for Differential Problems, T. Popoviciu Institute of Numerical Analysis, Cluj-Napoca, Romaina, 2007.

[10] E. H. Doha, W. M. Abd-Elhameed, and A. H. Bhrawy. New spectral-Galerkin algorithms for direct solution of high evenorder differential equations using symmetric generalized Jacobi polynomials. Collectanea Mathematica, vol. 64, no. 3, pp. 373-394, 2013

[11] E.H.Doha and A. H. Bhrawy. An efficient direct solver formultidimensional elliptic Robin boundary value problems using a Legendre spectral-Galerkin method. Computers Mathematics with Applications, vol. 64, no. 4, pp. 558571, 2012.

[12] A. H. Bhrawy1,2 andM. A. Alghamdi1. Approximate Solutions of Fisher's Type Equations with Variable Coefficients. Hindawi Publishing Corporation Abstract and Applied Analysis. Volume 2013, Article ID 176730, 10 pages. http://dx.doi.org/10.1155/2013/176730

[13] S. Tang and R.O. Weber. Numerical study of Fisher's equation by a Petrov-Galerkin finite element method, Jour. Austr. Math. Sot. 33, 27-38 (1991).

[14] T. Mavounugou and Y. Cerrault. Numerical study of Fisher's equation by Adomian's method, .Mathl. Comput. Modelling 19 (1), 89-95 (1994).

[15] Zhaosheng Feng. Traveling waves to a reaction diffusion equation. Discrete and continuous website: www.AIMSciences.org. Dynamical systems. pp. 382390. 